\title{
Association Studies for Yield and Its Component Traits in Basmati Genotypes of Himachal Pradesh, India
}

\author{
$\operatorname{Vinod~Kumar}^{1 *}$ and Dhirendra Singh ${ }^{2}$ \\ ${ }^{1}$ Department of Crop Improvement (Plant Breeding and Genetics), CSK, HP, Krishi \\ Vishvavidyalaya, Palampur-176062 (H.P.), India \\ ${ }^{2}$ Hill Agricultural Research and Extension Centre Dhaulakuan, CSK, HP, Krishi \\ Vishvavidyalaya, (H.P.), India \\ *Corresponding author
}

\begin{abstract}
A B S T R A C T
\section{Keywords}

Basmati rice, Correlation coefficients, Path

coefficient analysis, Residual effect

Article Info

Accepted:

10 February 2018 Available Online: 10 March 2018

An experiment was carried out with 30 basmati genotypes including 3 checks, were raised in randomized block design with three replications, to determine the inter-relationships among yield components and their direct and indirect contributions towards seed yield. The genotypic correlation coefficients were higher in magnitude than phenotypic correlation coefficients which revealed a strong inherent association between various characters under study and the phenotypic expression of correlations was lessened under the influence of environment. Grain yield per plant had a significantly positive correlation with spikelet per panicle, grains per panicle and fertility per cent. Grain length had positive and significant correlation with grain length after cooking, grain breadth and L: B ratio indicating typical basmati character that basmati varieties elongate length wise. Path analysis revealed that grains per panicle had a maximum direct effect on grain yield per plant followed by tillers per plant and 1000-grain weight. Therefore selection based on these characters either in combination or alone will help in improving basmati cultivars.

\section{Introduction}

Rice (Oryza sativa L.) is one of the major staple food crop of world especially of the most Asian countries like China, India, Pakistan, Bangladesh Vietnam and Korea. Rice is placed on second position in cereal production around the globe. More than $90 \%$ of the world's rice is grown and consumed in Asia, where $60 \%$ of the world's population lives. Rice accounts for between $35-60 \%$ of the caloric intake of three billion Asians. In India rice was grown on an area of 43.38

million hectares with a production of 104.32 million tonnes (Anonymous 2016). In Himachal Pradesh rice is a major kharif crop, next to maize and occupied an area of 72.5 thousand hectares with a production of 125.2 thousand tones with productivity of $17.3 \mathrm{q} / \mathrm{ha}$ (Anonymous 2016). Scented rice (Basmati) are known for their fine grains and cooking quality which includes longer and slender grain, higher grain elongation after cooking, low gelatinization temperature and intermediate amylose content. These quality characteristics coupled with high aroma add to
\end{abstract}


the value of basmati rice. In Himachal Pradesh, basmati rice is grown in a few isolated areas and the farmers mostly grow local cultivars which warrant development of high yielding scented rice varieties suitable for cultivation. Seed yield is a complex character which is highly influenced by interaction of various component traits and the environment. Therefore knowledge on nature of associations between yield and its component characters and their direct and indirect contributions on seed yield is necessary for efficient selection (Robinson et al., 1951). However when more characters are included in correlation study, the association become complex and correlation coefficient alone does not provide exact picture of relative importance of different characters and their direct and indirect influence on yield. In such situations, selection on the basis of direct and indirect effects is much more useful, using path analysis. Hence, the present investigation was undertaken to study the association among grain yield and its component characters along with the nature and extent of direct and indirect effects of yield components on the grain yield in basmati rice.

\section{Materials and Methods}

The present investigation was carried out with thirty genotypes of aromatic rice including three checks T-23, Hassan Serai and Kasturi (Table 1) in a randomized block design with three replications having a plot size of $3.0 \mathrm{~m} \times$ $1 \mathrm{~m}$, inter-row and inter-plant spacing of $20 \mathrm{~cm}$ and $15 \mathrm{~cm}$ respectively, at Rice and Wheat Research Centre, Malan during Kharif, 2013. All recommended cultural practices were followed to raise the experiment. Observations were taken from five random plants from each genotype and each replication on on characters such as days to 50 per cent flowering, plant height $(\mathrm{cm})$, tiller per plant $(\mathrm{cm})$, panicle length $(\mathrm{cm})$, spikelet per panicle, fertility percent, grains per panicle, 1000 grain weight (gm), grain yield per plant (gm) grain length $(\mathrm{mm})$, grain width $(\mathrm{mm})$, grain $\mathrm{L} / \mathrm{B}$ ratio, grain length after cooking $(\mathrm{mm})$, elongation ratio, amylose content (\%), gelatinization temperature (1-7 scale). In order to determine the inter-relationships among yield components and their direct and indirect contributions towards seed yield, correlation coefficients were calculated as per Al-Jibouri et al., 1958 and path coefficient were estimated as per Dewey and Lu, 1959.

\section{Results and Discussion}

The magnitude and nature of association of characters at genotypic and phenotypic levels are presented in table 2. In general, the estimates of genotypic correlation were higher in magnitude than the phenotypic correlation coefficient, indicating a fairly strong inherent interrelationship among the traits. At phenotypic and genotypic level, grain yield per plant had significant and positive correlation with spikelets per panicle $(\mathrm{rp}=0.5133 ; \mathrm{rg}=0.5761)$, grains per panicle $(\mathrm{rp}=0.5762 ; \mathrm{rg}=0.6310)$, and fertility per cent $(\mathrm{rp}=0.2124 ; \quad \mathrm{rg}=0.2199)$ and negative correlation with days to $50 \%$ flowering ( $\mathrm{rp}=-$ 0.2084 ; rg=-0.2400). These results were in consonance with the earlier reports of Sangeeta et al., (2001), Adil et al., (2007) Nayak et al., (2007), Akhtar et al., (2011), Bagati et al., (2016), Dhurai et al., (2016) and Behra et al., (2017) for grains per panicle and fertility per cent. It was observed that plant height was positively and significantly correlated with panicle length $(\mathrm{r} p=0.5019$; $\mathrm{rg}=0.5431)$, spikelets per panicle $(\mathrm{rp}=0.2797$; $\mathrm{rg}=0.2950)$, grains per panicle $(\mathrm{rp}=0.2813$; $\mathrm{rg}=0.2815), \quad \mathrm{L}: \quad \mathrm{B}$ ratio $\quad(\mathrm{rp}=0.3434$; $\mathrm{rg}=0.3813)$, amylose content $\quad(\mathrm{rp}=0.2864$; $\mathrm{rg}=0.3000)$ and gelatinization temperature $(\mathrm{rp}=0.2806 ; \mathrm{rg}=0.3251)$. Panicle length had significant and positive correlation with spikelets per panicle $(\mathrm{rp}=0.4935 ; \mathrm{rg}=0.5523)$, grains per panicle $(\mathrm{rp}=0.4581 ; \mathrm{rg}=0.5175)$, 
grain length ( $\mathrm{rp}=0.3035 ; \mathrm{rg}=0.3607)$ and $\mathrm{L}: \mathrm{B}$ ratio $(\mathrm{rp}=0.3209 ; \mathrm{rg}=0.3771)$. Spikelets per panicle was significantly and positively correlated with grains per panicle $(\mathrm{rp}=0.9066$; $\mathrm{rg}=0.9177), \quad$ grain length $\quad(\mathrm{rp}=0.2171$; $\mathrm{rg}=0.2226)$, and L: B ratio $(\mathrm{rp}=0.3687$; $\mathrm{rg}=0.3908$ ). Significant positive correlation of grains per panicle was noticed with fertility per cent ( $\mathrm{rp}=0.3050 ; \mathrm{rg} 0.2932)$ and L: B ratio $(\mathrm{rp}=0.3474 ; \mathrm{rg}=0.3828)$. Fertility per cent showed significantly positive correlation with elongation ratio $(\mathrm{rp}=0.2415 ; \mathrm{rg}=0.2738)$ and amylose content $(\mathrm{rp}=0.2472 ; \mathrm{rg}=0.2713)$. 1000 -grains weight was significantly and positively correlated with grain length $(\mathrm{rp}=0.6870 ; \quad \mathrm{rg}=0.7565), \quad$ grain breadth $(\mathrm{rp}=0.6734 ; \mathrm{rg}=0.7090)$ and grain length after cooking ( $\mathrm{rp}=0.2903 ; \mathrm{rg}=0.3177)$. Among the quality traits, grain length exhibited significantly positive association with grain length after cooking ( $\mathrm{rp}=0.3566 ; \mathrm{rg}=0.3664)$, grain breadth $(\mathrm{rp}=0.4873 ; \mathrm{rg}=0.5193)$ and $\mathrm{L}: \mathrm{B}$ ratio $(\mathrm{rp}=0.4057 ; \mathrm{rg}=0.3580)$.

Similar results were obtained by Zahid et al., (2006) for association between grain length and grain length after cooking, which is the typical basmati character as basmati varieties elongate length wise after cooking. L: B ratio had significantly positive correlation with grain length after cooking ( $\mathrm{rp}=0.2350$; $\mathrm{rg}=0.2322$ ). Grain length after cooking was significantly and positively correlated with elongation ratio $(\mathrm{rp}=0.6888 ; \mathrm{rg}=0.7041)$.

Table.1 List of materials used in the study

\begin{tabular}{|c|c|c|}
\hline Sr. No. & Designation & Parentage/Source \\
\hline 1 & HPR 2610 & Hassan Serai /T 23//IR 66295-36-2 \\
\hline 2 & HPR 2612 & Hassan Serai /T 23//IR 66295-36-2 \\
\hline 3 & HPR 2667 & Palampur Purple/ Kasturi \\
\hline 4 & HPR 2668 & Palampur Purple/ Kasturi \\
\hline 5 & HPR 2692 & Hassan Serai/T 23//IR 67011 \\
\hline 6 & HPR 2693 & Hassan Serai /T 23//IR 66295-36-2 \\
\hline 7 & HPR 2694 & Hassan Serai /T 23//IR 66295-36-2 \\
\hline 8 & HPR 2695 & Hassan Serai /T 23//IR 66295-36-2 \\
\hline 9 & HPR 2696 & Hassan Serai /T 23//IR 66295-36-2 \\
\hline 10 & HPR 2746 & Hassan Serai /T 23//IR 67011 \\
\hline 11 & HPR 2747 & Hassan Serai /T 23//IR 66295-36-2 \\
\hline 12 & HPR 2748-W & Hassan Serai /T 23//IR 66295-36-2 \\
\hline 13 & HPR 2749 & Hassan Serai /T 23//IR 66295-36-2 \\
\hline 14 & HPR 2751 & Hassan Serai /T 23//IR 66295-36-2 \\
\hline 15 & HPR 2753 & Hassan Serai /T 23//IR 66295-36-2 \\
\hline 16 & HPR 2754 & Hassan Serai /T 23//IR 66295-36-2 \\
\hline 17 & HPR 2755 & Hassan Serai /T 23//IR 66295-36-2 \\
\hline 18 & HPR 2757 & Hassan Serai /T 23//IR 66295-36-2 \\
\hline 19 & HPR 2761 & Hassan Serai/ Kasturi \\
\hline 20 & HPR 2850 & T 23/ Kasturi \\
\hline 21 & HPR 2853 & T 23/ Kasturi \\
\hline 22 & HPR 2854 & T 23/ Kasturi \\
\hline 23 & HPR 2855 & T 23/ Kasturi \\
\hline 24 & HPR 2857 & T 23/ Kasturi \\
\hline 25 & HPR 2861 & Palampur Purple/ Kasturi \\
\hline 26 & HPR 2863 & Palampur Purple/ Kasturi \\
\hline 27 & Sharbati & FRR 843-3/IR 38784-137-2-5//FRR843-3/IR 38787-26-2-2-3 \\
\hline 28 & $\mathrm{~T}-23$ & Pure line selection from a local cultivar \\
\hline 29 & Hassan Serai & Introduction from Iran \\
\hline 30 & Kasturi & Basmati 370/CRR 88-17-15 \\
\hline
\end{tabular}


Table.2 Estimates of phenotypic $(\mathrm{P})$ and genotypic $(\mathrm{G})$ correlation coefficient among different characters in basmati genotypes

\begin{tabular}{|c|c|c|c|c|c|c|c|c|c|c|c|c|c|c|c|c|}
\hline Traits & & $\begin{array}{c}\text { Days to } \\
\mathbf{5 0 \%} \\
\text { flowering }\end{array}$ & $\begin{array}{c}\text { Plant } \\
\text { height }\end{array}$ & $\begin{array}{c}\text { Tillers/ } \\
\text { plant }\end{array}$ & $\begin{array}{l}\text { Panicle } \\
\text { length }\end{array}$ & $\begin{array}{c}\text { Spikelets } \\
\text { per } \\
\text { panicle }\end{array}$ & $\begin{array}{c}\text { Grains } \\
\text { per } \\
\text { panicle }\end{array}$ & Fertility & $\begin{array}{l}1000- \\
\text { grains } \\
\text { weight }\end{array}$ & $\begin{array}{l}\text { Grain } \\
\text { length }\end{array}$ & $\begin{array}{c}\text { Grain } \\
\text { breadth }\end{array}$ & $\begin{array}{l}\text { L:B } \\
\text { ratio }\end{array}$ & $\begin{array}{l}\text { Grain } \\
\text { length } \\
\text { after } \\
\text { cooking }\end{array}$ & $\begin{array}{l}\text { Elongati } \\
\text { on ratio }\end{array}$ & $\begin{array}{l}\text { Amylose } \\
\text { content }\end{array}$ & GT \\
\hline \multirow{2}{*}{$\begin{array}{l}\text { Yield } \\
\text { /plant }\end{array}$} & rp & $-0.2084 *$ & 0.1086 & 0.1130 & 0.1124 & 0.5133* & $0.5762 *$ & $0.2124 *$ & 0.1350 & 0.0566 & 0.0767 & -0.0123 & 0.0151 & -0.0169 & 0.1103 & -0.0865 \\
\hline & rg & $-0.2400 *$ & 0.0744 & 0.1419 & 0.1599 & 0.5761* & $0.6310 *$ & 0.2199* & 0.1462 & 0.0194 & 0.0856 & -0.0553 & 0.0051 & 0.0067 & 0.1263 & -0.1059 \\
\hline \multirow{2}{*}{$\begin{array}{c}\text { Days to } \\
\mathbf{5 0 \%} \\
\text { flowering }\end{array}$} & rp & & 0.0122 & 0.0824 & 0.1528 & -0.0170 & -0.1447 & -0.3809 & $-0.2843^{*}$ & -0.1680 & -0.0903 & -0.0524 & -0.1229 & 0.0043 & -0.1047 & 0.0095 \\
\hline & rg & & 0.0197 & 0.0755 & 0.1523 & -0.0169 & -0.1519 & -0.4357 & $-0.3048 *$ & -0.1838 & -0.0970 & -0.0561 & -0.1409 & -0.0062 & -0.1096 & 0.0106 \\
\hline \multirow{2}{*}{$\begin{array}{l}\text { Plant } \\
\text { height }\end{array}$} & $\mathbf{r p}$ & & & -0.1408 & 0.5019* & $0.2797 *$ & $0.2813 *$ & 0.0505 & -0.0230 & 0.1162 & $-0.2110 *$ & 0.3434* & 0.1032 & 0.0060 & $0.2864 *$ & $0.2806 *$ \\
\hline & rg & & & $-0.2127^{*}$ & 0.5431* & $0.2950 *$ & $0.2815^{*}$ & 0.0190 & -0.0201 & 0.1204 & $-0.2324 *$ & 0.3813* & 0.1096 & 0.0105 & 0.3000* & $0.3251 *$ \\
\hline \multirow{2}{*}{$\begin{array}{c}\text { Tillers per } \\
\text { plant }\end{array}$} & rp & & & & $-0.2112 *$ & $-0.3628 *$ & $-0.2915^{*}$ & 0.1167 & -0.3825 & $-0.4040 *$ & -0.0344 & $-0.3418 *$ & -0.1510 & 0.1598 & -0.0403 & -0.1602 \\
\hline & rg & & & & $-0.3222 *$ & $-0.4901 *$ & $-0.3886 *$ & 0.2025 & -0.4746 & $-0.5678 *$ & -0.0620 & $-0.4619 *$ & $-0.2072 *$ & 0.2115 & -0.0631 & -0.1678 \\
\hline \multirow{2}{*}{$\begin{array}{c}\text { Panicle } \\
\text { length }\end{array}$} & rp & & & & & $0.4935^{*}$ & $0.4581 *$ & -0.0624 & 0.1687 & $0.3035^{*}$ & -0.0333 & 0.3209* & 0.1781 & $-\mathbf{- 0 . 0 7 0 9}$ & 0.0454 & 0.0144 \\
\hline & rg & & & & & 0.5523* & $0.5175^{*}$ & -0.0627 & 0.1651 & $0.3607^{*}$ & -0.0284 & 0.3771* & 0.1941 & -0.0947 & 0.0320 & 0.0287 \\
\hline \multirow{2}{*}{$\begin{array}{c}\text { Spikelets } \\
\text { per panicle }\end{array}$} & $\mathbf{r p}$ & & & & & & $0.9066 *$ & -0.1063 & 0.0686 & $0.2171 *$ & -0.1434 & $0.3687^{*}$ & 0.0056 & -0.1504 & 0.0070 & 0.1248 \\
\hline & rg & & & & & & 0.9177* & -0.0987 & 0.0755 & 0.2226* & -0.1509 & 0.3908* & -0.0057 & -0.1638 & 0.0100 & 0.1374 \\
\hline \multirow{2}{*}{$\begin{array}{l}\text { Grains per } \\
\text { panicle }\end{array}$} & $\mathbf{r p}$ & & & & & & & $0.3050 *$ & -0.0691 & 0.0356 & $-0.2869 *$ & 0.3474* & -0.0527 & -0.0663 & 0.0862 & 0.0993 \\
\hline & rg & & & & & & & 0.2932* & -0.0691 & 0.0430 & $-0.3016 *$ & 0.3828* & -0.0582 & $-\mathbf{- 0 . 0 7 7 6}$ & 0.0879 & 0.1019 \\
\hline \multirow[t]{2}{*}{ Fertility } & $\mathbf{r p}$ & & & & & & & & $-0.2670 *$ & $-0.4225 *$ & $-0.3209 *$ & -0.0594 & -0.0903 & 0.2415* & $0.2472 *$ & -0.0241 \\
\hline & rg & & & & & & & & $-0.3041^{*}$ & $-0.4519 *$ & $-0.3543 *$ & -0.0415 & -0.0758 & 0.2738* & 0.2713* & -0.0466 \\
\hline \multirow{2}{*}{$\begin{array}{l}\text { 1000- } \\
\text { grains } \\
\text { weight }\end{array}$} & rp & & & & & & & & & $0.6870 *$ & $0.6734 *$ & -0.0756 & 0.2903* & $-0.2421 *$ & -0.1861 & -0.1099 \\
\hline & rg & & & & & & & & & $0.7565^{*}$ & $0.7090 *$ & -0.0753 & $0.3177^{*}$ & $-0.2563 *$ & -0.1927 & -0.1252 \\
\hline \multirow{2}{*}{$\begin{array}{l}\text { Grain } \\
\text { length }\end{array}$} & rp & & & & & & & & & & $0.4873^{*}$ & $0.4057^{*}$ & $0.3566^{*}$ & $-0.4297^{*}$ & -0.1265 & -0.0198 \\
\hline & rg & & & & & & & & & & 0.5193* & 0.3580* & $0.3664 *$ & -0.4014 & -0.1334 & -0.0009 \\
\hline \multirow{2}{*}{$\begin{array}{c}\text { Grain } \\
\text { breadth }\end{array}$} & rp & & & & & & & & & & & $-0.5987 *$ & 0.0889 & $-0.2833^{*}$ & -0.2070 & -0.1757 \\
\hline & rg & & & & & & & & & & & $-0.6107 *$ & 0.1002 & $-0.2896^{*}$ & $-0.2195^{*}$ & -0.1781 \\
\hline \multirow[t]{2}{*}{ L:B ratio } & rp & & & & & & & & & & & & $0.2350 *$ & -0.0954 & 0.1043 & 0.1808 \\
\hline & rg & & & & & & & & & & & & $0.2322 *$ & $-\mathbf{0 . 0 5 2 0}$ & 0.1203 & $0.2100 *$ \\
\hline \multirow{2}{*}{$\begin{array}{c}\text { Grain } \\
\text { length after } \\
\text { cooking }\end{array}$} & rp & & & & & & & & & & & & & $0.6888 *$ & -0.1244 & -0.0826 \\
\hline & rg & & & & & & & & & & & & & 0.7041* & -0.1268 & -0.0903 \\
\hline \multirow{2}{*}{$\begin{array}{l}\text { Elongation } \\
\text { ratio }\end{array}$} & rp & & & & & & & & & & & & & & -0.0235 & -0.0674 \\
\hline & rg & & & & & & & & & & & & & & -0.0242 & -0.0904 \\
\hline \multirow{2}{*}{$\begin{array}{l}\text { Amylose } \\
\text { content }\end{array}$} & rp & & & & & & & & & & & & & & & -0.0285 \\
\hline & rg & & & & & & & & & & & & & & & -0.0244 \\
\hline
\end{tabular}


Table.3 Direct and indirect effects of component traits on yield at phenotypic at phenotypic and genotypic level

\begin{tabular}{|c|c|c|c|c|c|c|c|c|c|c|c|c|c|c|c|c|c|}
\hline Traits & & $\begin{array}{c}\text { Days to } \\
50 \% \\
\text { flowering }\end{array}$ & $\begin{array}{l}\text { Plant } \\
\text { height }\end{array}$ & $\begin{array}{l}\text { Tillers } \\
\text { per } \\
\text { plant }\end{array}$ & $\begin{array}{l}\text { Panicle } \\
\text { length }\end{array}$ & $\begin{array}{c}\text { Spikelet } \\
\text { per } \\
\text { panicle }\end{array}$ & $\begin{array}{c}\text { Grains } \\
\text { per } \\
\text { panicle }\end{array}$ & $\begin{array}{l}\text { Fertility } \\
\text { per cent }\end{array}$ & $\begin{array}{l}\text { 1000- } \\
\text { grains } \\
\text { weight }\end{array}$ & $\begin{array}{l}\text { Grain } \\
\text { length }\end{array}$ & $\begin{array}{c}\text { Grain } \\
\text { breadth }\end{array}$ & $\begin{array}{l}\text { L:B } \\
\text { ratio }\end{array}$ & $\begin{array}{c}\text { Grain } \\
\text { length } \\
\text { after }\end{array}$ & $\begin{array}{l}\text { Elongati } \\
\text { on ratio }\end{array}$ & $\begin{array}{l}\text { Amylose } \\
\text { content }\end{array}$ & G.T. & $\begin{array}{l}\text { Yield } \\
\text { per } \\
\text { plant }\end{array}$ \\
\hline \multirow{2}{*}{$\begin{array}{l}\text { Days to } 50 \% \\
\text { flowering }\end{array}$} & rp & 0.057 & 0.001 & 0.039 & -0.054 & 0.013 & -0.247 & 0.124 & -0.117 & 0.346 & -0.184 & -0.096 & -0.068 & -0.002 & -0.019 & -0.001 & $-0.208 *$ \\
\hline & rg & 0.058 & 0.000 & 0.089 & -0.037 & 0.038 & -0.542 & 0.517 & -0.242 & -0.832 & -0.089 & -0.052 & 0.946 & -0.044 & -0.053 & 0.002 & $-0.241 *$ \\
\hline \multirow[t]{2}{*}{ Plant height } & rp & 0.001 & 0.067 & -0.067 & -0.177 & -0.213 & 0.481 & -0.016 & -0.009 & -0.242 & -0.433 & 0.629 & 0.057 & 0.000 & 0.052 & -0.019 & 0.111 \\
\hline & rg & 0.001 & -0.020 & -0.251 & -0.132 & -0.671 & 1.004 & -0.023 & -0.016 & 0.549 & -0.215 & 0.347 & -0.734 & 0.039 & 0.144 & 0.050 & 0.072 \\
\hline \multirow{2}{*}{$\begin{array}{l}\text { Tillers per } \\
\text { plant }\end{array}$} & rp & 0.005 & -0.009 & 0.476 & 0.074 & 0.277 & -0.498 & -0.038 & -0.157 & 0.836 & -0.076 & -0.623 & -0.083 & -0.073 & -0.007 & 0.011 & 0.115 \\
\hline & rg & 0.004 & 0.004 & 1.179 & 0.078 & 1.115 & -1.386 & -0.240 & -0.377 & -2.575 & -0.055 & -0.421 & 1.379 & 1.492 & -0.030 & -0.026 & 0.141 \\
\hline \multirow[t]{2}{*}{ Panicle length } & rp & 0.009 & 0.033 & -0.101 & -0.352 & -0.377 & 0.783 & 0.020 & 0.069 & -0.628 & -0.072 & 0.588 & 0.099 & 0.033 & 0.008 & -0.001 & 0.111 \\
\hline & rg & 0.009 & -0.011 & -0.380 & -0.242 & -1.257 & 1.846 & 0.074 & 0.131 & 1.637 & -0.028 & 0.343 & -1.297 & -0.686 & 0.015 & 0.004 & 0.158 \\
\hline \multirow{2}{*}{$\begin{array}{l}\text { Spikelet per } \\
\text { panicle }\end{array}$} & rp & -0.001 & 0.019 & -0.173 & -0.174 & -0.763 & 1.550 & 0.035 & 0.028 & -0.448 & -0.298 & 0.675 & 0.003 & 0.069 & 0.001 & -0.008 & $0.515^{*}$ \\
\hline & rg & -0.001 & -0.006 & -0.578 & -0.134 & -2.276 & 3.274 & 0.117 & 0.060 & 1.010 & -0.142 & 0.356 & 0.034 & -1.164 & 0.005 & 0.021 & $0.576 *$ \\
\hline \multirow{2}{*}{$\begin{array}{l}\text { Grains per } \\
\text { panicle }\end{array}$} & rp & -0.008 & 0.019 & -0.139 & -0.161 & -0.692 & 1.709 & -0.099 & -0.028 & -0.072 & -0.597 & 0.635 & -0.029 & 0.030 & 0.016 & -0.007 & $0.577^{*}$ \\
\hline & rg & -0.009 & -0.006 & -0.458 & -0.125 & -2.088 & 3.568 & -0.348 & -0.055 & 0.193 & -0.284 & 0.348 & 0.386 & -0.549 & 0.042 & 0.016 & $0.631 *$ \\
\hline \multirow[t]{2}{*}{ Fertility } & rp & -0.022 & 0.003 & 0.056 & 0.022 & 0.081 & 0.521 & -0.325 & -0.110 & 0.875 & -0.664 & -0.111 & -0.050 & -0.110 & 0.045 & 0.002 & $0.213^{*}$ \\
\hline & rg & -0.025 & 0.000 & 0.239 & 0.015 & 0.225 & 1.046 & -1.188 & -0.241 & -2.056 & -0.333 & -0.038 & 0.509 & 1.945 & 0.131 & -0.007 & $0.222 *$ \\
\hline \multirow{2}{*}{$\begin{array}{l}\text { 1000-grains } \\
\text { weight }\end{array}$} & rp & -0.016 & -0.002 & -0.182 & -0.059 & -0.052 & -0.118 & 0.087 & 0.411 & -1.421 & 1.385 & -0.139 & 0.161 & 0.107 & -0.034 & 0.007 & 0.135 \\
\hline & rg & -0.018 & 0.000 & -0.560 & -0.040 & -0.172 & -0.247 & 0.361 & 0.793 & 3.433 & 0.661 & -0.069 & -2.119 & -1.768 & -0.093 & -0.019 & 0.143 \\
\hline \multirow[t]{2}{*}{ Grain length } & rp & -0.010 & 0.008 & -0.193 & -0.107 & -0.166 & 0.060 & 0.137 & 0.283 & -2.068 & 0.999 & 0.743 & 0.198 & 0.194 & -0.023 & 0.001 & 0.056 \\
\hline & rg & 0.039 & 0.048 & -0.619 & -0.037 & -0.456 & 0.401 & 0.788 & 0.650 & 0.389 & 0.532 & 0.576 & -0.950 & -1.326 & -0.014 & 0.000 & 0.021 \\
\hline \multirow[t]{2}{*}{ Grain breadth } & rp & -0.005 & -0.014 & 0.082 & 0.012 & 0.311 & -0.297 & 0.305 & 0.477 & -0.805 & 0.354 & -0.698 & 0.248 & 0.128 & -0.038 & 0.011 & 0.071 \\
\hline & rg & -0.006 & 0.005 & -0.069 & 0.057 & 0.347 & -1.037 & 0.424 & 0.563 & 2.353 & 0.731 & -0.557 & -0.654 & -1.99 & -0.056 & -0.027 & 0.084 \\
\hline \multirow[t]{2}{*}{ L:B ratio } & rp & 0.047 & 0.073 & -0.112 & -0.063 & -0.031 & 0.843 & 0.070 & 0.019 & -0.589 & -1.181 & 0.331 & 0.380 & 0.094 & 0.069 & 0.038 & -0.012 \\
\hline & rg & -0.003 & -0.007 & -0.495 & -0.091 & -0.839 & 1.415 & 0.050 & -0.060 & 1.673 & -0.569 & 0.610 & -1.549 & -0.329 & 0.108 & 0.032 & -0.054 \\
\hline \multirow{2}{*}{$\begin{array}{l}\text { Grain length } \\
\text { after cooking }\end{array}$} & rp & -0.007 & 0.007 & -0.072 & -0.013 & -0.005 & -0.090 & 0.029 & 0.119 & -0.689 & 0.178 & 0.430 & 0.403 & -0.260 & -0.022 & 0.005 & 0.013 \\
\hline & rg & -0.008 & -0.002 & -0.244 & -0.047 & 0.012 & -0.206 & 0.091 & 0.252 & 1.667 & 0.091 & 0.211 & -6.674 & 4.939 & -0.061 & -0.014 & 0.007 \\
\hline \multirow{2}{*}{$\begin{array}{l}\text { Elongation } \\
\text { ratio } \\
\end{array}$} & rp & 0.000 & 0.000 & 0.077 & 0.026 & 0.116 & -0.114 & -0.080 & -0.098 & 0.890 & -0.584 & -0.179 & 0.380 & -0.451 & -0.005 & 0.005 & -0.017 \\
\hline & rg & 0.000 & 0.050 & 0.300 & 0.524 & 0.877 & 0.221 & -0.279 & 0.300 & -1.325 & -0.220 & 0.001 & -1.439 & 0.530 & -0.015 & 0.485 & 0.010 \\
\hline \multirow{2}{*}{$\begin{array}{l}\text { Amylose } \\
\text { content }\end{array}$} & rp & -0.006 & 0.019 & -0.019 & -0.016 & -0.005 & 0.147 & -0.080 & -0.077 & 0.259 & -0.428 & 0.190 & -0.069 & 0.014 & 0.180 & 0.002 & 0.111 \\
\hline & rg & -0.006 & -0.006 & -0.074 & -0.008 & -0.023 & 0.314 & -0.322 & -0.153 & -0.599 & -0.206 & 0.109 & 0.847 & -0.224 & 0.482 & -0.004 & 0.127 \\
\hline \multirow[t]{2}{*}{ G.T. } & rp & 0.001 & 0.019 & -0.076 & -0.005 & -0.095 & 0.170 & 0.008 & -0.045 & 0.044 & -0.352 & 0.332 & -0.046 & 0.032 & -0.005 & -0.066 & -0.084 \\
\hline & rg & 0.001 & -0.006 & -0.198 & -0.007 & -0.313 & 0.364 & 0.055 & -0.099 & -0.012 & -0.161 & 0.192 & 0.602 & -0.666 & -0.012 & 0.154 & -0.106 \\
\hline
\end{tabular}

Significant at $5 \%$ level of significance

Residual effect: Phenotypic $=0.362$

Genotypic $=0.180$ 
Seed yield is a complex character which is highly influenced by interaction of various component traits and the environment. Compartmentalization of correlation coefficients into direct and indirect effects revealed the true nature of associations observed among various characters. The path coefficient analysis using phenotypic correlation coefficients among pair of characters depicting direct and indirect effects on seed yield showed the highest positive direct effect of grains per panicle $(\mathrm{rp}=1.709$; $\mathrm{rg}=3.568$ ) followed by tillers per plant $(\mathrm{rp}=0.476 ; \mathrm{rg}=1.179)$ and 1000 -grains weight $(\mathrm{rp}=0.411 ; \mathrm{rg}=0.793)$ at both phenotypic and genotypic level (Table 3). Concurrently, spikelets per panicle had indirect effect on grain yield via grains per panicle and L: B ratio at both phenotypic and genotypic level. Grains per panicle had indirect effect on grain yield via L: B ratio. At phenotypic level fertility per cent had indirect effect on grain yield via grains per panicle and grain length. At genotypic level fertility per cent had indirect effect on grain yield via grains per panicle and elongation ratio. In the present study, grains per panicle had direct effect on grain yield at phenotypic and genotypic level coupled with high positive correlation. The path coefficient analysis carried out at a phenotypic and genotypic levels showed similar trend in majority of traits. At both genotypic and phenotypic levels grains per panicle, tillers per plant, 1000-grains weight had highest positive direct effect on yield. So grains per panicle is an important parameter as it exhibit the high positive direct effect on yield at both genotypic and phenotypic levels coupled with high positive correlation and moderate to high value of residual effect. Similar results were obtained by Gravois and Helms (1992), Gazafrodi et al., (2006), Agahi et al., (2007) and Ritu (2008) as they also reported that grains per panicle and grain weight had positive direct effect on grain yield. Hossain et al., (2015) reported that effective tillers per plant had the positive direct effect on grain yield per plant followed by grains per panicle which is in accordance to present study.

From the present study it is concluded that spikelets per panicle, grains per panicle, and fertility percent showed positive and significant association with grain yield and also among themselves. Path analysis revealed that grains per panicle had a maximum direct effect on grain yield per plant followed by tillers per plant and 1000grain weight. Therefore simultaneous selection for these characters would result in improvement of yield. The genotypic (0.180) and phenotypic (0.362) residual effect were considerably low indicating the significant contribution of the characters taken for study towards yield per plant.

\section{References}

Adil, J., Muhammad, A., Ejaz, A and Zadid, M.A. 2007. Genotypic and phenotypic correlation among various plant traits in rice hybrids (Oryza sativa L.). Science International Lahore 19(1): 287-290.

Agahi, K., Fotokian, M.H and Farshadfar, E. 2007. Correlation and path coefficient analysis for some yield related traits in rice genotypes (Oryza sativa L). Asian Journal of Plant Sciences 6: 513-517.

Akhtar, N., Nazir, M.F., Rabnawaz, A., Mahmood, T., Safdar, M.E., Asif, M and Rehman, A. 2011, Estimation of heritability, correlation and path coefficient analysis in fine grain rice (Oryza sativa L.), The Journal of Animal and Plant Sciences, 21(4):660664

Al-Jibouri, H.A., Millar, P.A and Robinson, H.P. 1958. Genotypic and environmental variance and co-variance in upland cotton cross of inter-specific origin. Agronomy Journal 50: 633-636. 
Anonymous. 2016. Food and Agriculture Organization; http://faostat.fao.org.

Anonymous. 2016. Statistical Outline of Himachal Pradesh 2015-16. Department of Economics and Statistics, Directorate of land records, Government of Himachal Pradesh.

Bagati, S., Singh, A.K., Salgotra, R.K., Bhardwaj, R., Sharma, M., Rai, S.K and Bhat, A. 2017. Genetic variability, heritability and correlation coefficients of yield and its component traits in basmati rice (Oryza sativa L.). SABRAO Journalof Breeding and Genetics 48 (4): 445-452

Behera, M., Monalisa, S.P., Bastia, D.N and Kulkarn, CC. 2017. Correlation and Path Analysis Studies in Aerobic Rice. International Journal of Current Microbiology and Applied Sciences 6(8): 2851-2856

Dewey, D.R and Lu, K.H. 1959. A correlation and path analysis of components of crested wheat grass seeds population. Agronomy Journal 51(8): 515-518.

Dhurai, S.Y., Reddy, D.M and Ravi, S. 2016. Correlation and path analysis for yield and quality characters in rice (Oryza sativa L.). Rice Genomics and Genetics 7(4): 1-6

Gazafrodi, A.A., Honarnegad, R., Fotokian, M.H and Alami, A. 2006. Study of correlations among agronomic traits and path analysis in rice (Oryza sativa L). Journal of Science and Technology of Agriculture and Natural Resources 10: 99-107.

Gazafrodi, A.A., Honarnegad, R., Fotokian, M.H and Alami, A. 2006. Study of correlations among agronomic traits and path analysis in rice (Oryza sativa L). Journal of Science and Technology of Agriculture and Natural Resources 10: 99-107

Gravois, K.A and Helms, R.S. 1992. Path analysis of rice yield and yield components as affected by seedling rate. American Society of Agronomy Journal 84(1): 1-4.

Hossain, S., Haque, M.M.D and Rahman, J. 2015. Genetic variability, correlation and path coefficient analysis of morphological traits in some extinct local aman rice (Oryza sativa L.). Journal of Rice Research 4(1): 1-7

Nayak, A.R. 2007. Heritability and correlation in scented rice. Indian Agriculturist 51(1): 9-12

Ritu. 2008. Genetic analysis of morphological, biochemical and molecular traits of some novelty rice of Himachal Pradesh. Ph.D. Thesis. Department of Plant Breeding and Genetics, CSKHPKV Palampur.

Robinson, H.F., Comstock, R.E and Harvey, P.H. 1951. Genotypic and phenotypic correlations in corn and their implications in selection. Agronomy Journal 43: 282-287.

Sangeeta, M., Giri, D.G., Jahagirdar, S.W and Giri, M.D. 2001. Correlation studies in upland rice. Annals of plant Physiology 14(1)196-197.

Zahid, Akhte, M.A., Sabar, M., Zaheen, M and Tahir. 2006. Correlation and path analysis studies of yield and economic traits in Basmati rice (Oryza sativa L.). Asian Journal of Plant Sciences 5(4):643-645.

\section{How to cite this article:}

Vinod Kumar and Dhirendra Singh. 2018. Association Studies for Yield and Its Component Traits in Basmati Genotypes of Himachal Pradesh, India. Int.J.Curr.Microbiol.App.Sci. 7(03): 1243-1249. doi: https://doi.org/10.20546/ijcmas.2018.703.147 